\title{
Structural, Morphological and Magnetic Studies on Sol-Gel Multi-Doped $\mathrm{BiFeO}_{3}$ Compounds
}

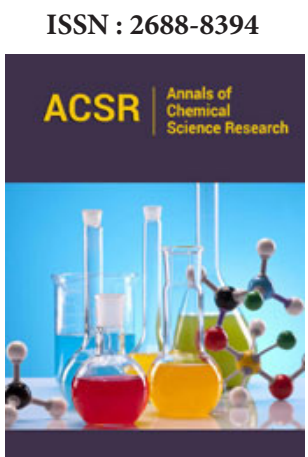

*Corresponding author: Costa BFO, Department of Physics, University of Coimbra, Portugal

Submission: 漈 August 25, 2020

Published: 留September 04, 2020

Volume 2 - Issue 3

How to cite this article: Benali $A$ Bejar M, Dhahri E, Valente MA, Costa BFO. Structural, Morphological and Magnetic Studies on Sol-Gel MultiDoped Bifeo3 Compounds. Ann Chem Sci Res. 2(3). ACSR. 000536. 2020. DOI: 10.31031/ACSR.2020.02.000536

Copyright@ Costa BFO, This article is distributed under the terms of the Creative Commons Attribution 4.0 International License, which permits unrestricted use and redistribution provided that the original author and source are credited.

\section{Benali $\mathrm{A}^{1,2,3}$, Bejar $\mathrm{M}^{2}$, Dhahri $\mathrm{E}^{2}$, Valente $\mathrm{MA}^{3}$ and Costa $\mathrm{BFO}^{1 *}$}

${ }^{1}$ University of Coimbra, CFisUC, Department of Physics, Coimbra, Portugal

${ }^{2}$ Laboratoire de Physique Appliquée, Faculté des Sciences, Université de Sfax, Sfax, Tunisia

${ }^{3}$ I3N, Physics Department, University of Aveiro, Aveiro, Portugal

\begin{abstract}
The $\mathrm{Bi}_{0.8} \mathrm{Er}_{0.1} \mathrm{Ba}_{0.1} \mathrm{Fe}_{0.96} \mathrm{R}_{0.02} \mathrm{Cr}_{0.02} \mathrm{O}_{3}\left(\mathrm{R}=\mathrm{Mn}^{3+}\right.$ and $\left.\mathrm{Co}^{2+}\right)$ compounds were prepared by the Sol-Gel method and sintered at $800{ }^{\circ} \mathrm{C}$ for 2 hours. Both compounds present a rhombohedral structure with R3C space group. The nano-size criteria is confirmed for both compounds. The compound with $\mathrm{Mn}^{3+}$ ions presents the higher saturation magnetization and the higher amount of ferromagnetic contributions.
\end{abstract}

Keywords: Multiferroics; Sol-gel; Doped $\mathrm{BiFeO}_{3}$; Magnetization; Transmission electron microscopy

\section{Introduction}

Materials showing a simultaneous coexistence of electric and magnetic ordering have high interesting properties allowing them to be used in several application arrears especially in next-generation memory devices such as in electric fields control magnetism. These materials are known as multiferroic materials. Among these materials, bismuth ferrite with the general formula $\mathrm{BiFeO}_{3}$ (BFO) stands up, because it is one of the limited materials being simultaneously magnetic and strongly ferroelectric at room temperature $[1,2]$. The pure BFO presents an anti-ferromagnetism with Neel temperature around 750K. Indeed, the weak magnetic behaviour and leakage currents are still the drawbacks for its application in the case of novel magnetoelectric effects. However, it was reported that the substitution in A or/ and B-sites with suitably doped ions is deemed to be a compromising way to modulate and enhance the ferromagnetic and ferroelectric properties at room temperature of magnetic materials [3-6].

Considering the previous, the discussion of structural, morphological, magnetic and dielectric properties of BFO compound prepared by sol-gel and with the substitution of $10 \%$ of $\mathrm{Er}^{3+}$ and $10 \%$ of $\mathrm{Ba}^{2+}$ in $\mathrm{A}$-site of $\mathrm{BiFeO}_{3}$ compound simultaneously with $4 \%$ of $\mathrm{Mn}^{3+}$ or $\mathrm{Co}^{2+}$ and $\mathrm{Cr}^{3+}(2 \%$ each one) in its B-site was done [7-9].

\section{Results and Discussion}

\section{Structural and morphological studies}

X-ray diffractograms of the $\mathrm{Bi}_{0.8} \mathrm{Er}_{0.1} \mathrm{Ba}_{0.1} \mathrm{Fe}_{0.96} \mathrm{Co}_{0.02} \mathrm{Cr}_{0.02} \mathrm{O}_{3} \quad$ (BEBFCC) and $\mathrm{Bi}_{0.8} \mathrm{Er}_{0.1} \mathrm{Ba}_{0.1} \mathrm{Fe}_{0.96} \mathrm{Mn}_{0.02} \mathrm{Cr}_{0.02} \mathrm{O}_{3}$ (BEBFMC) compounds were indexed to bismuth ferrite material with the formula $\mathrm{BiFeO}_{3}$ (JCPDS file no. 71-2494) with rhombohedral structure, $\mathrm{R} 3 \mathrm{C}$ space group, which confirms well the formation of the desired perovskite multiferroic material. Furthermore, minor impurity peaks are also evident in the XRD patterns of both compounds. Xpert-hight score was used to prove that these peaks correspond to the $\mathrm{Bi}_{2} \mathrm{Fe}_{4} \mathrm{O}_{9}$ and $\mathrm{Bi}_{25} \mathrm{FeO}_{40}$ phases. The formation of these secondary phases during the synthesis of undoped BFO and cations substituted BFO is almost unavoidable accordingly to similar studies $[10,11]$. 
The average crystallite size, estimated from XRD patterns using Scherrer formula was found to be around $68 \mathrm{~nm}$ and $35 \mathrm{~nm}$ in the case of BEBFCC and BEBFMC, respectively. The composition and purity of the prepared compounds were determined by energy dispersive X-ray microanalysis. The surface morphology was investigated by scanning electron microscopy and the micrographs showed that the grains are irregular and non-uniformly distributed as in the case of the pure BFO compound [7]. The particle size determination was done by transmission electron microscopy using image-J software, as shown in Figure 1 for BEBFMC. According to a Lorentzian adjustment of the particles size distribution, a value of $57 \mathrm{~nm}$ was found. One can deduce that each particle has 2 crystallites in average. The nanosized criteria of the prepared compounds was confirmed.
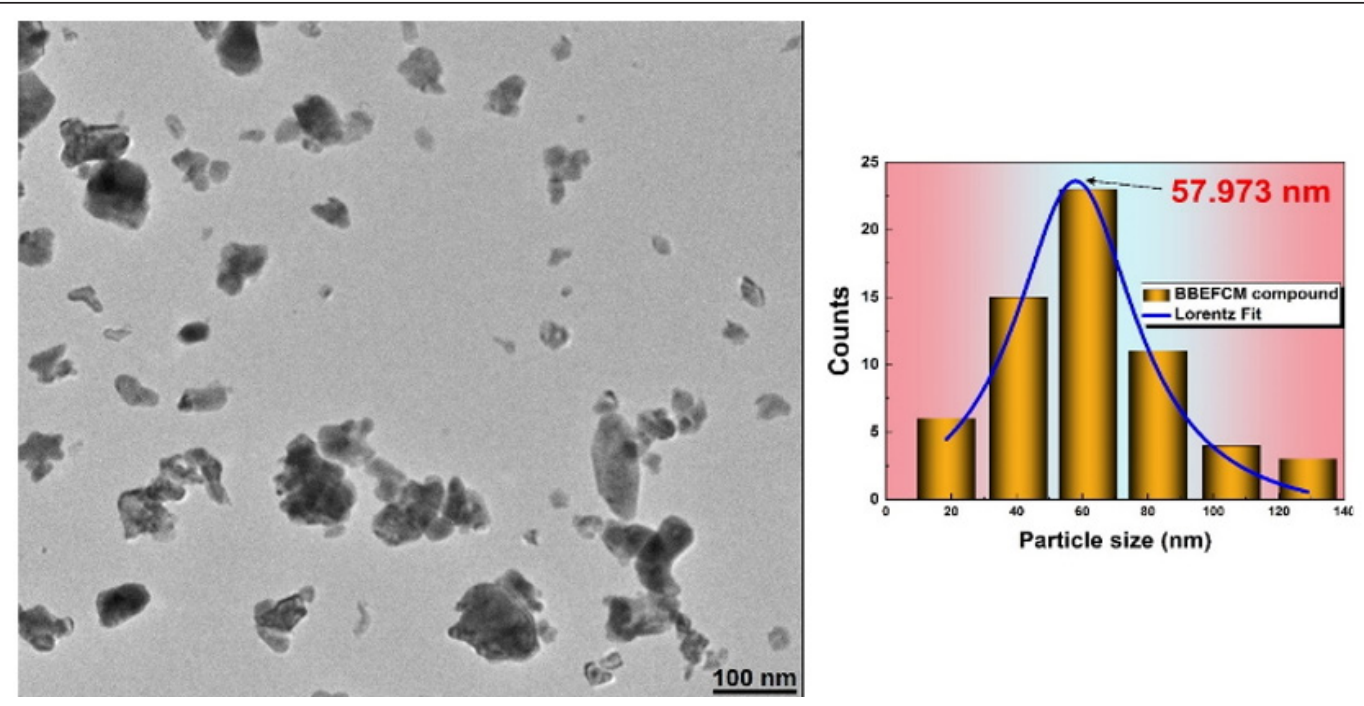

Figure 1: TEM images and particles size distribution of the BEBFMC compound.

\section{Magnetic studies}

The temperature dependence of the magnetization under 0.05T of the multiferroic BEBFMC and BEBFCC are shown in Figure 2. One can notice that the magnetization of the BEBFMC compound is higher than that of BEBFCC. Clearly, the magnetization of both compounds, rises when decreasing temperature and shows an inflection point at around $569 \mathrm{~K}$ and $575 \mathrm{~K}$ for BEBFMC and BEBFCC, respectively. This temperature is attributed to the FerromagneticParamagnetic phase transition known as the Curie Weis $\left(\mathrm{T}_{\mathrm{C}}\right.$ ) temperature. It is important to mention that the $\mathrm{T}_{\mathrm{C}}$ temperature of the pure $\mathrm{BiFeO}_{3}$ compound was found to be higher than 750K [12], so the utility of the substitution in both A and B sites was confirmed to decrease the $\mathrm{T}_{\mathrm{C}}$ temperature, especially in the case of $\mathrm{Mn}$ ions. The room temperature hysteresis $(\mathrm{M}(\mathrm{H})$ ) loops of the BEBFMC and BEBFCC compounds are shown in Figure 2, respectively. As one can see, in both compounds, the magnetization occurs in two different behaviours; first region where the magnetization increases very sharply with increasing magnetic field, while at high magnetic fields the magnetization is more-or-less saturated and exhibits a linear behaviour. For pure BFO, the M-H curves exhibit an almost unsaturated straight line which proves that it has a G-type Antiferromagnetic (AFM) with canting of $\mathrm{Fe}^{3+}$ ions [13]. Both behaviours at high and low magnetic fields, confirm the existence of a competition between ferromagnetic (FM) and antiferromagnetic (AFM) interactions due to the substitution in $\mathrm{A}$ and B-sites of the BFO compound. This magnetic competition enhances the total magnetization in the studied compound. The saturated magnetization $\mathrm{M}_{\mathrm{S}}$ of the BEBFMC and BEBFCC compounds were found to be around 6.8 and $5.5 \mathrm{emu} / \mathrm{g}$, respectively, at room temperature which is more than seventeen times larger than that of the pure BFO compound (about $0.3 \mathrm{emu} / \mathrm{g}$ ) [14,15]. This enhancement of magnetization is due essentially due to the FM interactions occurring with the substitutions of both A and B-sites.

The M-H loops have been modulated to quantify theoretically the FM and the AFM contributions, using the following equation:

$M(H)=\left\{2 \frac{M_{F M}^{S}}{\pi} \tan ^{-1}\left(\left(\frac{H \pm H_{c i}}{H_{c i}}\right) \tan \left(\frac{\pi \times M_{F M}^{R}}{2 \times M_{F M}^{S}}\right)\right)\right\}+\{\chi H\}$

where $M_{F M}{ }^{S}, M_{F M}{ }^{R}, H_{c i}$ are the ferromagnetic saturation magnetization, remnant magnetization, intrinsic coercive field, respectively. The first term in this equation is ascribed to the irreversible component of magnetization, coming from the ferromagnetic contribution (FM). The second one is due to the antiferromagnetic contribution (AFM) and shows a linear behaviour with the applied magnetic field $[16,17]$. The simulated ferromagnetic and antiferromagnetic contributions at room temperature for both compounds are shown in Figure 2. The resulted fitting parameters are listed in Table 1. From the fitted parameters values, it is concluded that for BEBFMC compound, the AFM contributions are around $34.48 \%$ of the total magnetization, while the FM part presents $65.62 \%$, which confirms the domination of the FM contribution over the AFM one. As conclusion, the enhancement of the magnetization in BEBFMC is due especially to the ferromagnetic contribution originated from the substitutions in A and B-sites, while for BEBFCC compound, the AFM contribution dominates the total magnetization (61.3\%) over the FM. 

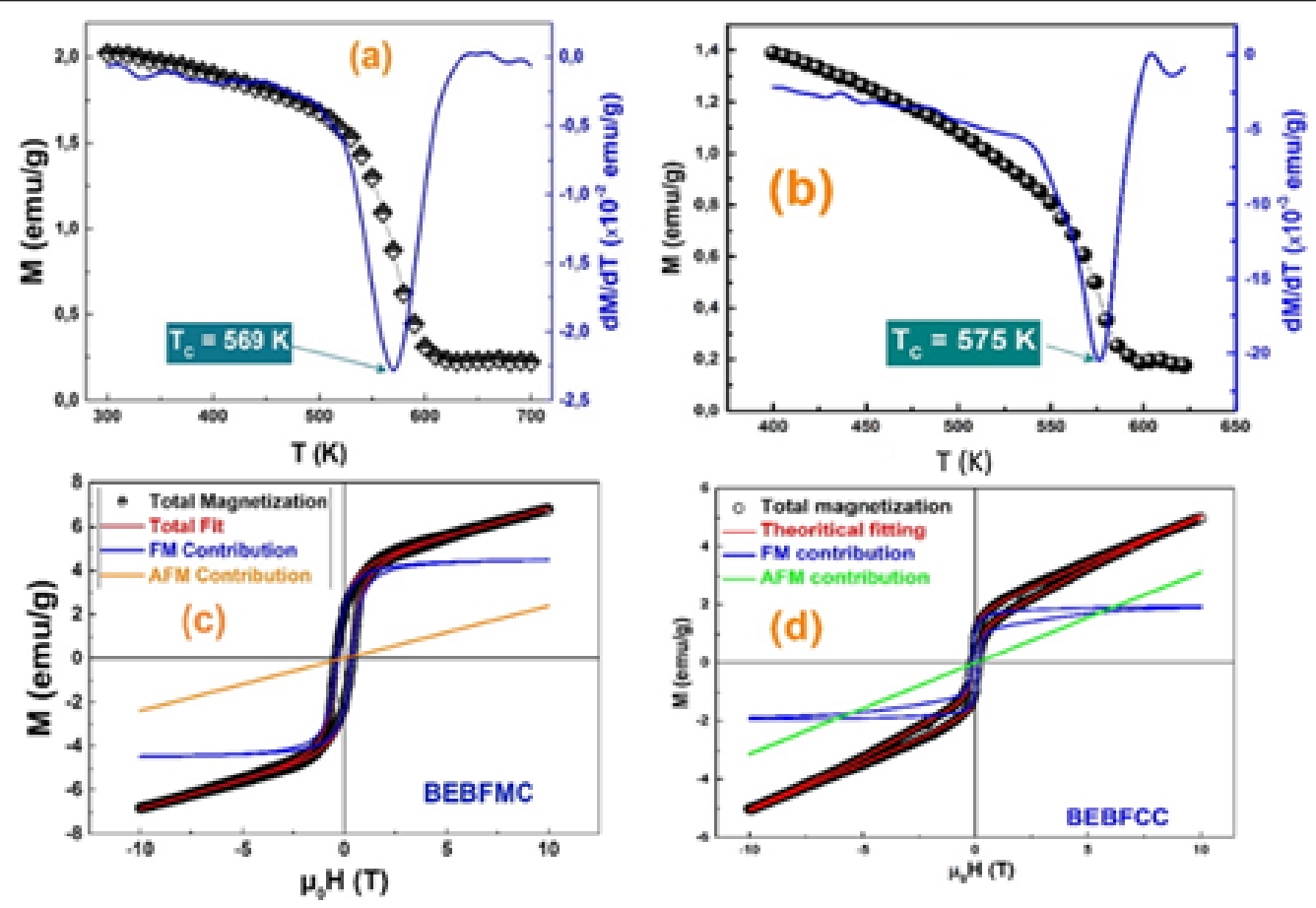

Figure 2: (a) and (b) Magnetization vs. temperature and its first derivation of the BEBFMC and BEBFCC compounds, respectively. (c) and (d) Fit results of the Hysteresis loop at room temperature of the BEBFMC and BEBFCC compounds, respectively.

Table 1: Parameters extracted from fitting the magnetization hysteresis loops of the compounds.

\begin{tabular}{|c|c|c|c|c|}
\hline \multirow{2}{*}{ Sample } & Antiferromagnetic Contribution & \multicolumn{3}{|c|}{ Ferromagnetic Contribution } \\
\hline \multirow{2}{*}{ BEBFMC } & $\chi\left(\mathrm{emu} / \mathrm{g}^{*} \mathrm{~T}\right)$ & $\left.\mathrm{H}_{\mathrm{Cl}} \mathrm{T}\right)$ & $\mathrm{M}_{\mathrm{FM}} \mathrm{S}(\mathrm{emu} / \mathrm{g})$ & $\mathrm{M} \mathrm{F}_{\mathrm{FM}} \mathrm{R}(\mathrm{emu} / \mathrm{g})$ \\
\cline { 2 - 5 } & 0.371 & 0.415 & 6.838 & 2.756 \\
\cline { 2 - 5 } & 0.313 & 0.202 & 1.943 & 1.039 \\
\hline
\end{tabular}

\section{Conclusion}

The utility of multi doping the $\mathrm{BiFeO}_{3}$ system was confirmed. When introducing a low concentration of Erbium and Barium in A-site with a low amount of Chromium and (Manganese or Cobalt) in B-site, the total magnetization rises more than seventeen times compared to undoped $\mathrm{BiFeO}_{3}$ compound. The $\mathrm{Mn}^{3+}$ ions in B-site enhances the amount of the FM contribution in the compound leading to a rise in magnetization.

\section{Acknowledgement}

CFisUC is supported by national funds from FCT-Fundação para a Ciência e a Tecnologia, I.P., within the project UID/04564/2020. Access to TAIL-UC facility funded under QREN-Mais Centro Project No. ICT_2009_02_012_1890 is gratefully acknowledged.

\section{References}

1. Zhao T, Scholl A, ZavaLiche F, Lee K, Barry M, et al. (2006) Electrical control of antiferromagnetic domains in multiferroic $\mathrm{BiFeO}_{3}$ films at room temperature. Nat Mater 5(10): 823-829.

2. Pabst GW, Martin LW, Chu YH, Ramesh R (2007) Leakage mechanisms in $\mathrm{BiFeO}_{3}$ thin films. Applied Physics Letters 90: 072902.
3. Yan F, Lai MO, Lu L, Zhu TJ (2010) Enhanced multiferroic properties and valence effect of Ru-doped $\mathrm{BiFeO}_{3}$ thin films. Journal of Physical Chemistry C 114(15): 6994-6998.

4. Kharel P, Talebi S, Ramachandran B, Dixit A, Naik VM, et al. (2009) Structural, magnetic, and electrical studies on polycrystalline transitionmetal-doped $\mathrm{BiFeO}_{3}$ thin films. Journal of Physics: Condensed Matter 21(3): 036001.

5. Xing W, Ma Y, Bai YL, Zhao (2015) Enhanced ferromagnetism of Erdoped $\mathrm{BiFeO}_{3}$ thin films derived from rhombohedral-to-orthorhombic phase transformations. Mater Lett 161: 216-219.

6. Peng L, Deng HM, Tian JJ, Ren Q, et al. (2013) Influence of Co doping on structural, optical and magnetic properties of $\mathrm{BiFeO}_{3}$ films deposited on quartz substrates by sol-gel method. Appl Surf Sci 268: 146-150.

7. Benali A, Melo BMG, Prezas PR, Bejar M, Dhahri E, et al. (2019) Structural, morphological, Raman and ac electrical properties of the multiferroic so-gel made $\mathrm{BiErBaFeCrCoO}_{3}$ material. J Alloys and Comp 775: 304-315.

8. Benali A, Bejar M, Dhahri E, Hlil E, Graça MPF, et al. (2019) Assessment of the critical behavior in the multiferroic $\mathrm{BiBaErFeCrCoO}_{3}$ material, multisubstitutional effect on magnetic and Mössbauer properties, submitted.

9. Benali A, Bejar M, Dhahri E, Valente MA, Costa BFO (2007) International conference on multifunctional materials and their Applications, Monastir, Tunisia. 
10. Dang NV, Thanh TD, Hong LV, Lam VD, Phan TL (2011) Structural, optical and magnetic properties of polycrystalline $\mathrm{BaTi}_{1}-\mathrm{Fe}_{\mathrm{x}} \mathrm{O}_{3}$ ceramics. J Appl Phys 110(4): 043914.

11. Phadan SK, Roul BK (2011) Effect of Gd doping on structural, electrical and magnetic properties of $\mathrm{BiFeO}_{3}$ electroceramic, J Phys Chem Solids 72(10): 1180-1187.

12. Chang LY, Tu CS, Chen PY, Chen CS, Schmidt VH, et al. (2016) Raman vibrations and photovoltaic conversion in rare earth doped (Bi0.93R0.07) $\mathrm{FeO}_{3}$ (RE=Dy, Gd, Eu, Sm) ceramics. Ceram Int 42: 834-842.

13. Lebeugle D, Colson D, Forge A, Viret M, Bataille AM, et al. (2008) Electricfield-induced spin flop in $\mathrm{BiFeO}_{3}$ single crystals at room temperature. Phys Rev Lett 100: 227602-1.
14. Vijayasundaram SV, Suresh G, Mondal RA, Kanagadurai R (2016) Substitution-driven enhanced magnetic and ferroelectric properties of $\mathrm{BiFeO}_{3}$ nanoparticles. J of Alloys and Compd 658: 726-731.

15. Mao V, Wang X, Chu L, Zhu Y, Wang Q et al. (2016) Simultaneous enhancement of magnetic and ferroelectric properties in Dy and Cr codoped $\mathrm{BiFeO}_{3}$ nanoparticles. Phys Chem Chem Phys 18: 6399-6405.

16. Shah LR, Zhu H, Wang WG, Ali B, Zhu T, et al. (2010) Effect of Zn interstitials on the magnetic and transport properties of bulk Co-doped ZnO. J Phys D: Appl Phys 43(3): 035002.

17. Kumar P, Shankhwar N, Srinivasan A, Kar M (2015) Oxygen octahedra distortion induced structural and magnetic phase transitions in $\mathrm{Bi}_{1-}$ ${ }_{x} \mathrm{Ca}_{\mathrm{x}} \mathrm{Fe}_{1-\mathrm{x}} \mathrm{Mn}_{\mathrm{x}} \mathrm{O}_{3}$ ceramics. J Appl Phys 117(19): 194103.

For possible submissions Click below: 